InnOvaciOnes de NegOciOs 17(33): 1-20

(C) 2020 UANL, Impreso en México (ISSN: 2007-1191)

Recepción: 7 Noviembre de 2019 Aceptación: 8 Enero de 2020

\title{
La administración de la deuda pública de los gobiernos locales desde la perspectiva del modelo de la nueva gestión pública \\ (Public debt management in local governments from the new public management perspective)
}

\author{
Salvador Velázquez Caltzonzit \\ Universidad Autónoma de Querétaro \\ salvauaq@gmail.com
}

\begin{abstract}
The objective of this study is to analyze the impact of the implementation of the management model called the New Public Management in the management of public debt and the financial risk in the local governments in Mexico. The analysis was carried out from a descriptive and correlational perspective. Focusing on a first moment on the administrative logic of that managerial model that privileges the economy, efficiency and effectiveness, bringing organizational changes that have adjusted the structures, functions, strategies and organizational culture in the Public Administration; and in a second moment, in the analysis of the evolution of the balances of financial obligations and the legal framework that formally regulates the public debt in the states and municipal entities. The study seeks to test the research hypotheses that was formulated as follows: The implementation of the management model called The New Public Management has caused the accelerated increase in the level of indebtedness, putting public finances at risk in local governments in Mexico. The presentation of the hypothesis arises from the observation of the accelerated growth of balances of financial obligations in the states and municipal entities, as well as the treatment promoted by the legislative agenda in relation to public debt, which consisted in the implementation of various administrative and financial reforms focused on containing the excessive increase in the balances of financial obligations, without having empirical evidence that such purpose has been achieved until now.
\end{abstract}

Key words: New Public Management, public debt, financial risk, local governments.

JEL: H6, H60, H61, H62, H63.

Resumen: El objetivo del presente estudio consiste en analizar el impacto de la implementación del modelo gerencial denominado la Nueva Gestión Pública en la administración de la Deuda Pública y el riesgo financiero en los Gobiernos Locales de México. El análisis se llevó a cabo desde una perspectiva descriptiva y correlacional.

La administración de la deuda pública de los gobiernos 
Centrándose en un primer momento en la lógica administrativa de dicho modelo gerencial que privilegia a la economía, eficiencia y la eficacia; trayendo consigo cambios organizacionales que han ajustado a las estructuras, funciones, estrategias y cultura organizacional en la Administración Pública; y en segundo momento, en el análisis de la evolución de los saldos de las obligaciones financieras y el marco jurídico que regula formalmente la deuda pública en las entidades Estatales y Municipales. El estudio busca probar la hipótesis de investigación que se formuló de la siguiente manera: La implementación del modelo gerencial denominado la Nueva Gestión Pública provocando el incremento acelerado del nivel de endeudamiento, poniendo en riesgo las finanzas públicas en los Gobiernos locales en México. El planteamiento de la hipótesis surge de la observación del crecimiento acelerado de los saldos de las obligaciones financieras en las entidades Estatales y Municipales, así como del tratamiento promovido por la agenda legislativa en relación a la Deuda Pública, que consistió en la implementación de diversas reformas administrativas y financieras enfocadas en contener el incremento desmedido de los saldos de las obligaciones financieras, sin que hasta el momento exista evidencia empírica de dicho propósito.

Palabras clave: Nueva Gestión Pública, Deuda Pública, Riesgo financiero, Gobiernos Locales

\section{Introducción}

Dentro del marco de Reforma Administrativa del Estado, la Administración Pública en México vive la proliferación e implementación de modelos organizacionales de corte gerencial como lo es la Nueva Gestión Pública en adelante NGP, la cual privilegia a la eficiencia como principio básico y eje rector. Dicho modelo gerencial tiene como argumento central, el que la calidad y eficiencia se logran si se cuenta con un sector público menos centralizado, que compita y demuestre la obtención de resultados, mediante mediciones de desempeño, dejando en un segundo plano el cumplimiento de normas (Magallón, 2008).

La NGP de acuerdo con Magallón (2004), se ha legitimado bajo el argumento de que la Administración Pública tiene regulaciones que convierten a las decisiones en letárgicas. Mientras que la NGP contiene enfoques de decisión y pasión por la eficiencia y eficacia, promoviendo la necesidad de ganar más que gastar, convirtiendo al gobierno burocrático en gobierno empresarial y competitivo. En este sentido Christensen y Laegreid (2001), mencionan que un sistema de Gobierno empresarial eficiente y

\section{Velázquez, S.}


racional no ha demostrado ser fructífero cuando tiene que enfrentarse a la vida cotidiana del sector público.

Torres y Juárez (2016), indican que la NGP es un modelo gerencial más destacado en la Administración Pública, aparece como respuesta a la crisis de los Gobiernos, en el momento que se evidenció el estancamiento que la burocracia en el Estado. En este sentido, Torres y Juárez señalan que el paradigma que imperaba en ese momento era el de un Gobierno que actuaba de manera unilateral, autoritaria, ineficaz e incompetente para satisfacer las necesidades de la población. De tal forma que en dicho contexto se implementó el modelo de la NGP en los países occidentales debido a que el modelo de gestión tradicional o Gobierno benefactor ya no resolvía los problemas sociales en términos de eficiencia, eficacia y legitimidad.

A partir de dichas discusiones enunciadas en párrafos anteriores en la cual se plantean tensiones y paradojas que se observan en el modelo de la NGP, surge el interés por investigar sobre cuál habría de ser el papel del Estado en las nuevas condiciones sociales, su ámbito, tipo de acción; particularmente en el terreno de desarrollo económico y social. Es decir, qué puede y qué no puede hacer y cómo debe hacerlo (Aguilar, 2006).

De tal forma que, el análisis del impacto de la NGP en la administración de la deuda pública de los Gobiernos locales en México, busca demostrar que su lógica administrativa basada en la eficiencia y aunado la excesiva discrecionalidad en el uso de las facultades de los funcionarios públicos durante la gestión de los Gobiernos Neoliberales, han traído consigo un desmedido incremento de las obligaciones financieras poniendo en riesgo la sustentabilidad de las finanzas públicas en los Estados y municipios (Auditoria Superior de la Federación, 2011).

Considerando el contexto de la discusión planteada en párrafos anteriores en torno a las tensiones y paradojas sobre la implementación el objetivo de este estudio consiste en llevar a cabo el análisis del impacto de la NGP en el incremento desmedido de los niveles de las obligaciones financieras y del riesgo financiero en los Gobiernos locales en México.

\section{La administración de la deuda pública de los gobiernos}




\section{Marco Teórico}

\section{La nueva Gestión Pública}

El origen de la NGP tuvo lugar en países anglosajones durante los años ochenta, debido a las crisis económicas y a las recurrentes críticas a los gobiernos por su notable ineficiencia. En dicho periodo se modifican las estructuras, rutinas y prioridades de los empleados de los Gobiernos. La NGP durante la llegada del neoliberalismo, origina que los gobiernos de países como los Estados Unidos de América, declaren que el gobierno no era la solución sino el problema. Mientras que en el Reino Unido se nombraban a los directores de empresas privadas para dirigir al sector público con el propósito de hacerlo más eficiente (Cejudo, 2011).

La pérdida de credibilidad en el Gobierno acuerdo con García (2007), proviene de las exigencias de la ciudadanía obligándose a actuar aplicando principios de eficiencia y eficacia. Las demandas son más relevantes debido al déficit público en la mayor parte de los países del mundo, que ha impactado en la construcción de políticas públicas. Dichas politicas políticas se circunscriben al ámbito de las actuaciones de la administración en sus diferentes órdenes territoriales, que han llevado a diseñar un nuevo sistema operativo público, una nueva filosofía de actuación con base en la NGP.

En un entorno de ideas económicas y políticas favorables al mercado, la NGP aparece como la respuesta a los problemas sociales en muchos países. La implementación de la NGP en México, se da dentro de un marco de cambios políticos y económicos inducidos por los Gobiernos Neoliberales, promoviendo el aumento de la eficacia y la reducción de costos, orientado a mejorar sustancialmente al sector público, mientras que para los Gobiernos que padecían presiones fiscales y demandas sociales planteaba respuestas prometedoras (Cejudo, 2011).

Aguilar (2006), menciona que la reforma administrativa en la administración pública es una respuesta obligada a los problemas de operación del Gobierno al déficit fiscal de los Estados y a la baja calidad en los servicios públicos, así como a la implementación de políticas económicas erróneas. Evidenciando la equivocación e ineficacia gubernamental en los campos de la gestión administrativa y financiera en la asignación y uso de recursos públicos.

\section{Velázquez, S.}


Ante esta situación, en México se promueven reformas financieras y administrativas de corte gerencial enfocadas a contener los niveles elevados de endeudamiento en los Estados y municipios, modificando el marco jurídico que regula al financiamiento en los tres niveles de Gobierno y en el mecanismo de contratación de sus financiamientos. Dichos acontecimientos relevantes en la Administración Pública son descritos a continuación.

\section{El contexto de las reformas financieras en México}

El crecimiento significativo del saldo de la deuda pública de los Gobiernos locales, se ha convertido en tema central de la agenda legislativa, debido a que pone en riesgo la sostenibilidad de las finanzas públicas de las entidades federativas y municipios (Auditoria Superior de la Federación, 2011). La prioridad se debe al elevado nivel de endeudamiento que representa comprometer una proporción del $78.2 \%$ de las Participaciones Federales en adelante PF, en el cuarto trimestre del 2017. Esta situación es relevante, si se considera la amplia dependencia que tienen los Estados y municipios sobre dichos recursos federales para pago de las obligaciones financieras ante la baja recaudación de recursos propios, en adelante SHCP (Secretaria de Hacienda y Crédito Público, 2018).

La Auditoria Superior de Fiscalización, en adelante ASF (2017), menciona que las causas de endeudamiento en los Gobiernos locales son diversas, adicionalmente a las nuevas alternativas de financiamiento que disponen de garantía y fuente de pago con cargo a las PF, son los factores que han influido significativamente en el creciente endeudamiento. Por otra parte, la ASF menciona que existen causas de carácter estructural que han contribuido al acelerado crecimiento del nivel de deuda, entre las que destacan: la elevada dependencia de los recursos Federales y baja aportación de recursos propios provenientes de la recaudación fiscal de los Gobiernos locales, creciente déficit presupuestario debido a su política de gasto local, regulación débil, opacidad y nula rendición de cuentas sobre el ejercicio de los recursos obtenidos a través del financiamiento.

Debido a la diversidad de causas de carácter estructural que han incrementado los niveles de deuda pública en los Gobiernos locales, la agenda legislativa ha diseñado y promovido una serie de reformas financieras y fiscales, introduciendo modelos gerenciales y fiscales enfocados

\section{La administración de la deuda pública de los gobiernos}


en contener el nivel de deuda. De tal forma que, uno de los cambios relevantes que trajo consigo las reformas financieras y fiscales en el año de 1995, consistió en la modificación del marco jurídico que regula al financiamiento en los tres niveles de Gobierno, acentuado la inclusión de medidas prudenciales en la administración de la deuda pública que se observaron específicamente en los cambios de la normatividad de: La Ley de Coordinación Fiscal, Reglamento del artículo $9^{\circ}$ de la Ley de Coordinación fiscal, Reglas de capitalización de la banca, Sistemas para el ahorro para el retiro, Reforma al Artículo 115 Constitucional y Ley del Mercado de Valores.

A este respecto, se destaca la reforma a la legislación fiscal que se observa en el Artículo $9^{\circ}$. de la Ley de Coordinación Fiscal publicada en el Diario Oficial de la Federación (1995), en la cual se modificó de manera fundamental el mecanismo mediante el cual los Estados y Municipios contrataran sus financiamientos y cuyos principales cambios se explican en la tabla 1.

Tabla 1. Cambios al artículo $9^{\circ}$ de la Ley de Coordinación Fiscal

\begin{tabular}{|c|c|}
\hline LCF anterior & LCF actual \\
\hline $\begin{array}{l}\text { Los pagos de las obligaciones } \\
\text { garantizadas con la afectación de } \\
\text { participaciones se realizan siguiendo el } \\
\text { orden cronológico de su inscripción y } \\
\text { estarán a cargo de la SHCP. }\end{array}$ & $\begin{array}{l}\text { Los pagos de las obligaciones garantizadas } \\
\text { con la afectación de participaciones estarán a } \\
\text { cargo de los Estados y Municipios, de acuerdo } \\
\text { con los mecanismos y sistemas de registro } \\
\text { establecidos en sus leyes estatales de deuda. } \\
\text { En todo caso las entidades federativas } \\
\text { deberán contar con un registro único de } \\
\text { obligaciones y empréstitos, así como publicar } \\
\text { en reforma periódica su información con } \\
\text { respecto a los registros de deuda. }\end{array}$ \\
\hline
\end{tabular}

Fuente: Diario Oficial de la Federación (1995).

El nuevo mecanismo de pago requirió de la implementación de un Fideicomiso maestro que está garantizado por el flujo de PF, presentes y futuras cuyos objetivos consistieron en recibir la titularidad del porcentaje acordado con los Gobiernos locales para realizar el pago de las deudas a los acreedores y llevar a cabo el registro de las deudas.

Por otra parte, la aprobación de la Ley de Disciplina Financiera de las Entidades Federativas y Municipios en adelante LDFEFM, que de acuerdo con el Congreso de la Unión (2016), el objetivo se centra en promover las

\section{Velázquez, S.}


finanzas públicas sostenibles en dichas entidades, con base en la implementación de reglas de disciplina financiera, el uso responsable de la deuda pública, fortalecimiento de la trasparencia y la observación de cinco componentes principales que se relacionan en la tabla 2.

Dichas reformas financieras se enfocaron en el establecimiento de la certidumbre, estabilidad, fortalecimiento y modernización del Sistema Financiero Mexicano, modificando fundamentalmente los mecanismos mediante los cuales los Estados y Municipios contratarían sus financiamientos en el futuro (Diario oficial de la Federación, 2016).

Tabla 2. Componentes de la LDFEFM

\begin{tabular}{|c|c|}
\hline Componente & Definición \\
\hline $\begin{array}{l}\text { Reglas de disciplina } \\
\text { hacendaria y financiera }\end{array}$ & $\begin{array}{l}\text { Incentivan finanzas públicas sanas en gobiernos locales } \\
\text { mediante principios de responsabilidad hacendaria. }\end{array}$ \\
\hline Sistemas de alertas & $\begin{array}{l}\text { El sistema alerta cualquier riesgo ante los endeudamientos } \\
\text { de las entidades federativas y los municipios y obliga a } \\
\text { cumplir con convenios de responsabilidad hacendaria. }\end{array}$ \\
\hline $\begin{array}{l}\text { Contratación de deuda y } \\
\text { obligaciones }\end{array}$ & $\begin{array}{l}\text { Se garantiza que se contrate deuda al menor costo } \\
\text { financiero y de manera transparente con fines que tengan } \\
\text { beneficios. }\end{array}$ \\
\hline Deuda estatal garantizada & $\begin{array}{l}\text { El Gobierno de la República dará su aval crediticio con el } \\
\text { propósito de que los estados y municipios accedan a un } \\
\text { financiamiento más barato. }\end{array}$ \\
\hline Registro público único & $\begin{array}{l}\text { El registro sirve para inscribir y transparentar los } \\
\text { financiamientos y obligaciones }\end{array}$ \\
\hline
\end{tabular}

Fuente: Diario Oficial de la Federación (2016).

Las reformas administrativas y financieras cuentan con el soporte del marco jurídico que regula formalmente la Deuda Pública en los Gobiernos locales, el cual se conforma por una serie de normas prudenciales que se observan en el marco jurídico que establece la Constitución Política de los Estados Unidos Mexicanos, el cual se aborda en el siguiente apartado.

El marco jurídico en la administración de la deuda publica

Las normas prudenciales en materia financiera que rigen a las deudas públicas de los Gobiernos estatales y municipales se observan dentro del marco jurídico que establece La Constitución Política de los Estados Unidos Mexicanos, específicamente en el mandato del Artículo 117 Fracción

\section{La administración de la deuda pública de los gobiernos}


Octava que menciona el siguiente texto: los Estados y Municipios no podrán contraer obligaciones o empréstitos sino cuando se destinen a inversiones públicas productivas, inclusive los que contraigan los organismos descentralizados y empresas públicas, conforme a las bases que establezcan las legislaturas en una ley y por los conceptos y hasta por los montos que las mismas fijen anualmente en los respectivos presupuestos.

El texto del mandato Constitucional ha sido interpretado y ejecutado de diferente forma en las respectivas constituciones y leyes de deuda pública en los Gobiernos locales. A este respecto se destaca que 25 de las 32 Constituciones y Leyes de deuda de Gobiernos locales en México, incluyeron normas similares o más rígidas que las que menciona la Constitución Política Federal de acuerdo con Aregional (2005), las cuales versan sobre el destino del financiamiento, los límites de endeudamiento y especialmente la restricción del uso de los recursos de la deuda en el gasto corriente y la fuente de financiamiento. En dicho contexto, resulta pertinente analizar la evolución de los saldos de las obligaciones financieras y reflexionar sobre el impacto del modelo denominado la NGP en la administración de la deuda pública en los Gobiernos locales.

\section{Metodología}

El diseño metodológico del presente estudio busca obtener evidencia empírica que permita poner a prueba a la hipótesis de investigación planteada de la siguiente forma. Hi: La implementación del modelo NGP en la administración de la deuda pública incrementó los niveles de las obligaciones financieras y el riesgo financiero de los Gobiernos locales en México. El diseño metodológico toma las aportaciones del paradigma cuantitativo implementando estudios descriptivos y correlacionales. De tal forma que a continuación, se muestra la serie de pasos metodológicos requeridos para el presente estudio:

1) Análisis descriptivo: Se realiza una descripción de las tendencias de los saldos de deuda de Gobiernos locales considerando su relación con Participaciones Federales Ramo 28 y Producto Interno Bruto por sus siglas PIB en adelante, tomando para tal fin los periodos de análisis comprendidos entre los años 2003 al 2017 (Anderson, Sweeney, \& Williams, 2008).

\section{Velázquez, S.}


2) Análisis de correlación: Se realiza a través del modelo de regresión lineal simple con el propósito de conocer cómo ha impactado la implementación de la NGP en el incremento de las obligaciones financieras y riesgo financiero en los Gobiernos locales en México (Anderson et al., 2008). El análisis requirió en un primer momento de la prueba estadística basada en el cálculo del coeficiente de correlación entre dos variables de razón, con el propósito de medir la fuerza y dirección de una relación lineal.

Por otra parte, conocer si las dos variables están correlacionadas, sin llegar a establecer una relación de causalidad. Y posteriormente se procedió a calcular el coeficiente de determinación que permitió conocer la proporción de la variación en $\mathrm{Y}$ explicada por $\mathrm{X}$ en términos de porcentaje, implementado el siguiente modelo estadístico:

$$
\begin{array}{ll}
Y_{i}=\beta_{0}+\beta_{1} x_{1} & \text { Donde: } \\
& Y_{i}=\text { Variable dependiente } \\
& \beta_{0}=\text { Intercepto calculado } \\
& \beta_{1}=\text { Pendiente calculada } \\
& x_{1}=\text { Variable independiente de relación }
\end{array}
$$

\section{Resultados}

Estudio descriptivo: La situación de los saldos de las obligaciones financieras considerando su relación con PF Ramo 28, PIB y tiempo.

La evolución de la Deuda Pública en las últimas décadas en las Entidades Federativas y municipios de México, han mostrado máximos históricos en los saldos de sus obligaciones financieras. Se puede observar en la Figura 1 que existen tres cortes significativos a lo largo de la curva en diferentes periodos, los cuales describen tendencias y montos máximos de las obligaciones financieras dentro del periodo 2003-2017 (Secretaria de Hacienda y Crédito Público, 2018).

El comportamiento muestra una tendencia alcista significativa de los saldos de las obligaciones financieras en los últimos ocho años, situación que se considera pertinente analizar desde la perspectiva de la fuente de repago

\section{La administración de la deuda pública de los gobiernos}


basada en las PF del Ramo 28, tema que será abordado en apartados posteriores de este documento.

Figura 1. Nivel de endeudamiento de Estados y municipios en México

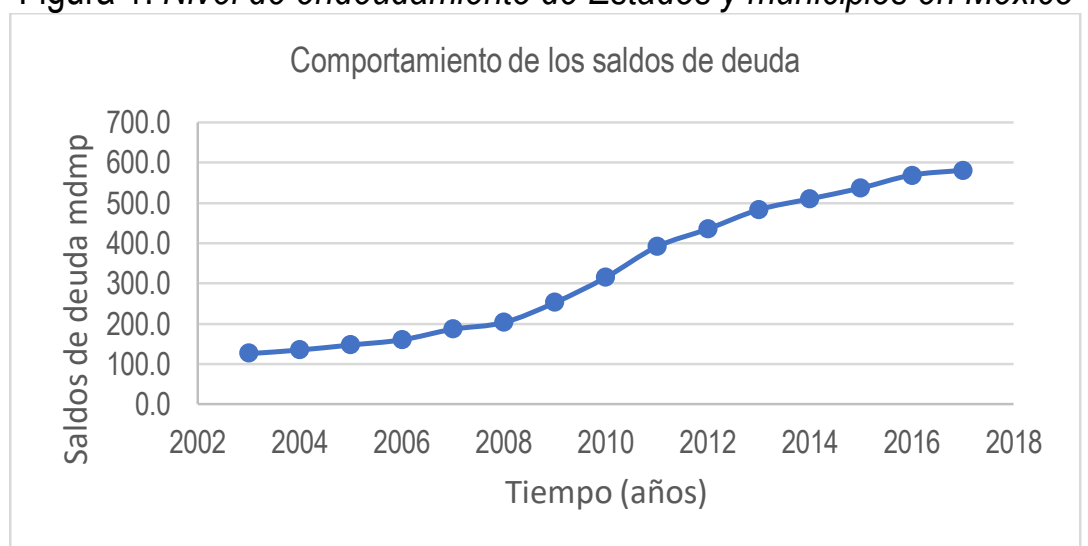

Fuente: elaboración propia con base en SHCP (2018)

Con relación al primer corte de tiempo, se considera importante mencionar que durante el periodo 2003-2006, la tendencia alcista de los saldos de las obligaciones financieras mostró una situación estable. En dicho periodo se observa que los saldos de deuda pública se habían podido contener, debido a que el saldo de deuda de 2003 a 2006 paso de $\$ 125.9$ mil mdp a $\$ 160.1$ mil mdp, según se muestra en la tabla 3.

Tabla 3. Saldos de las obligaciones financieras en gobiernos locales (mmdp)

\begin{tabular}{ccc}
\hline Periodo & Saldo de obligaciones financieras & Incremento porcentual \\
\hline $2003-2006$ & \$125.9 a \$160.1 & $27.2 \%$ \\
$2007-2012$ & $\$ 186.5$ a $\$ 404.4$ & $116.8 \%$ \\
$2013-2017$ & \$482.8 a $\$ 580.6$ & $20.2 \%$ \\
\hline
\end{tabular}

Fuente: elaboración propia con base en SHCP (2018).

Por lo que corresponde al segundo corte del tiempo, se observa un incremento altamente significativo en el saldo de la deuda de los gobiernos locales, debido a que en el año 2012 el saldo fue $\$ 404,4$ mil mdp pasando a $\$ 217.9$ mil mdp lo que representa una tasa de crecimiento de $116.8 \%$.

En el tercer corte de tiempo, comprende el periodo del 2013 al 2017, que se caracterizó por mostrar incrementos más significativos de deuda,

\section{Velázquez, S.}


alcanzando niveles máximos históricos de $\$ 580.6$ mil mdp. Este momento representó ser el punto de mayor riesgo en la sustentabilidad de las finanzas públicas de los Gobierno locales, por el incremento de los saldos en $361.2 \%$. Con base en la descripción del crecimiento acelerado de la deuda pública anteriormente descrito, el presente estudio aborda el análisis de la relación de los saldos de deuda a PF del Ramo 28, debido a que dichos recursos económicos están destinados al pago de las obligaciones financieras contratadas, permitiendo comprender en esta relación el riesgo financiero potencial para los Gobiernos locales que se puede observar en la figura 2.

Figura 2. Deuda como porcentaje de los ingresos por Participaciones Federales Ramo 28

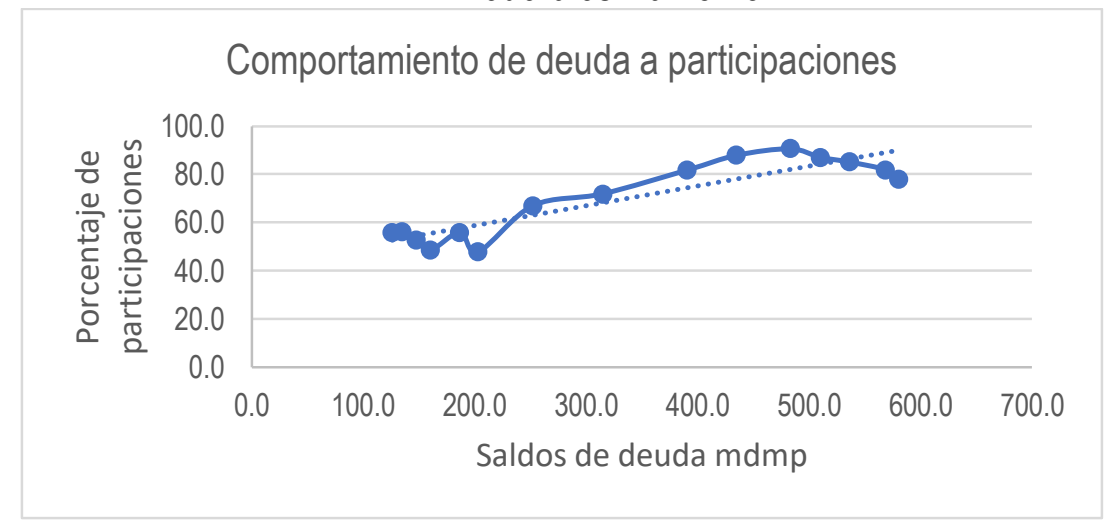

Fuente: elaboración propia con base en SHCP (2018).

El análisis de las cifras de la relación Deuda a PF del Ramo 28, muestran un incremento significativo a partir del año 2009 cuyo indicador fue de $67.1 \%$ con tendencia alcista, se destaca que el punto más alto se observa en el año 2013, alcanzado su máximo valor en el orden de 90.7\%. En este sentido se puede interpretar que, si se considera que las PF del Ramo 28 son la fuente de pago para las obligaciones crediticias en los Gobiernos locales, la cobertura de pago por cada 100 pesos de PF, \$ 90.7 pesos serian aplicados para liquidar el total de la deuda, viéndose cada vez más comprometidos los recursos.

Cabe señalar que aún y cuando las cifras de dicho indicador en el año 2017 son de 78.2\%, la situación financiera en los Gobiernos locales se considera preocupante, si se considera específicamente que dichos

\section{La administración de la deuda pública de los gobiernos}


Gobiernos se caracterizan por tener una baja recaudación tributaria y por su alta dependencia de PF Ramo 28 para desarrollar programas sociales, de inversión en obra pública y el gasto corriente.

El segundo indicador macroeconómico de interés de estudio, lo constituye la deuda como porcentaje del PIB, debido a que permite conocer la proporción con relación al PIB (Secretaria de Hacienda y Crédito Público, 2018), el valor del indicador se ubicó en 3.0\% en el año 2017, incrementando $2 \%$ con respecto al año 2002, tal y como se describe en la figura 3.

Figura 3. Deuda como porcentaje del producto interno Bruto

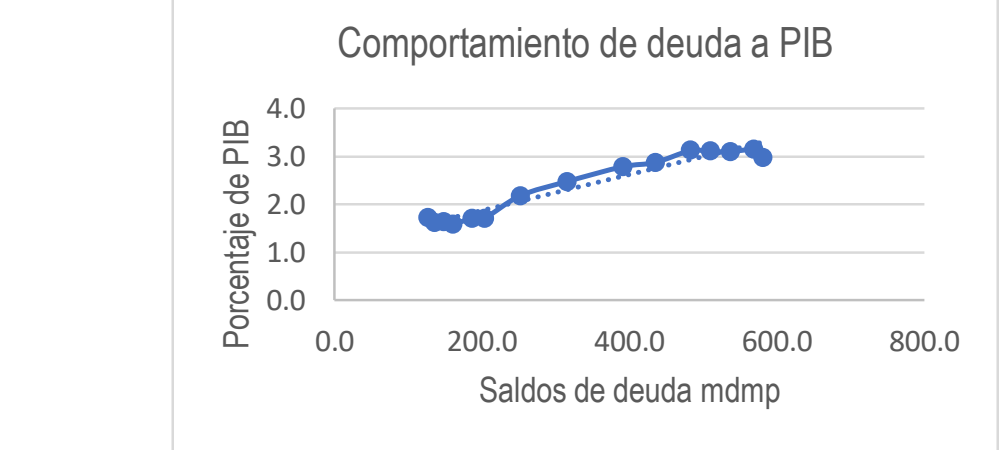

Fuente: elaboración propia con base en SHCP (2018).

Este análisis debe ser tomado con reserva, debido a que el monto total de la deuda pública de los Gobiernos locales con respecto al PIB indica un nivel de $3 \%$ en 2017 , interpretándose que dicho nivel no representaría un riesgo para las finanzas públicas para los Gobiernos locales. Sin embargo, el riesgo potencial para las finanzas públicas se debe observar desde la perspectiva de sus fuentes de ingresos, tales como PF del Ramo 28 y Recursos propios. Aclarando que el pago no está a cargo de las economías de los Gobiernos locales en términos de PIB. Por lo tanto, esta medición no es recomendable.

Estudio correlacional: Considera el análisis de las relaciones de la deuda como porcentaje de los ingresos por Participaciones Federales Ramo 28 y la deuda como porcentaje del producto interno Bruto y tiempo respectivamente.

\section{Velázquez, S.}


a) Coeficientes de correlación y determinación Deuda a PF Ramo 28

Con base en los valores de los coeficientes calculados que se describen en la tabla 4, se puede observar que la correlación entre las variables saldos de deuda a PF presentan una $r=.9064$ significándose como correlación positiva muy alta. Es decir, ambas variables aumentan simultáneamente.

Tabla 4. Coeficientes de correlación y determinación saldos de deuda a Participaciones Federales

\begin{tabular}{cc}
\hline Tipo de coeficiente & Valor calculado \\
\hline Coeficiente de correlación $(r)$ & .9064 \\
Coeficiente de determinación $\left(\mathrm{r}^{2}\right)$ & .8112 \\
\hline
\end{tabular}

Fuente: elaboración propia con base en SHCP (2018).

Y por lo que corresponde al cálculo del coeficiente determinación, arrojo un valor de $\mathrm{r}^{2}=.8112$ significando que la proporción de la variación en $Y$ saldos de deuda se explica por efecto de X, es decir, los niveles de PF Ramo 28 , aumentan en la medida en que se incrementa los saldos de deuda, según se describe en la figura 4.

Figura 4. Coeficiente de determinación de saldos de deuda a Participaciones

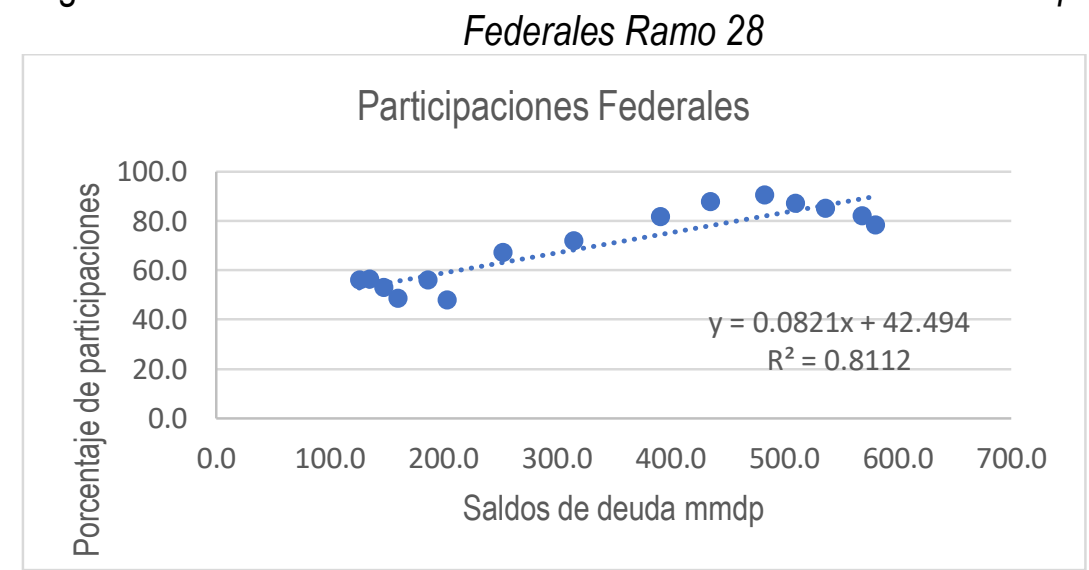

Fuente: elaboración propia con base en SHCP (2018).

\section{La administración de la deuda pública de los gobiernos}


b) Coeficientes de correlación y determinación de saldos de deuda a PIB

El análisis de correlación lineal aplicado en las variables saldos de deuda como porcentaje de PIB, arrojó el valor de r=.9719 que se muestra en la tabla 5, el cual indica la existencia de una correlación positiva muy alta, en donde ambas variables aumentan en forma simultánea.

De acuerdo con la Figura 4. se presenta la ecuación de regresión lineal simple dada de acuerdo con las siguientes consideraciones:

$$
y_{i}=42.492+0.0821 x
$$

$y_{i}=$ Participaciones federales

Donde:

$\beta_{0}=$ Intercepto

$\beta_{1}=$ Pendiente calculada

$x_{1}=$ Deuda Pública

Al respecto, se reconoce que el nivel de las PF Ramo 28 será de 42.49 millones de pesos cuando la deuda tenga un valor igual a cero. Mientras que en la medida que los saldos de la deuda incrementen en una unidad, las PF destinadas a la cobertura de la deuda incrementarán en 0.0821 mil mdp, por lo cual implica un grado mayor de compromiso de los recursos federales para el pago de la misma.

Tabla 5. Coeficientes de correlación y determinación saldos de deuda como porcentaje de PIB

\begin{tabular}{cc}
\hline Tipo de coeficiente & Valor calculado \\
\hline Coeficiente de correlación $(r)$ & .9719 \\
\hline Coeficiente de determinación $\left(r^{2}\right)$ & .9447 \\
\hline
\end{tabular}

Fuente: elaboración propia con base en SHCP (2018).

El coeficiente determinación calculado para dichas variables, arrojo un valor de $r^{2}=.9447$ significando que la proporción de la variación en $Y$ que corresponde a porcentaje de PIB se explica por efecto de X, es decir, la proporción de PIB aumentan en la medida en que se incrementa el nivel de deuda, permitiendo observar que el $94.47 \%$ de los incrementos de los porcentajes de PIB se vieron afectados por las variaciones de los saldos de deuda, según se describe en la figura 5 .

\section{Velázquez, S.}


Figura 5. Saldos de deuda como porcentaje de PIB

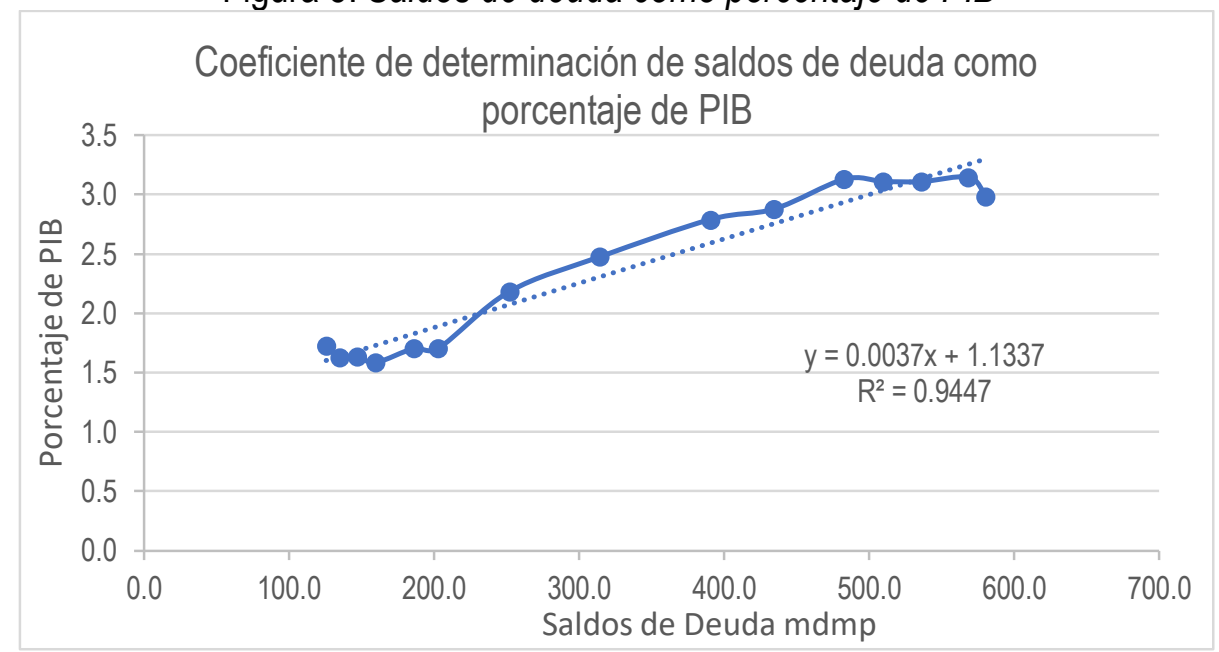

Fuente: elaboración propia con base SHCP (2018).

De acuerdo con la Figura 5. se presenta la ecuación de regresión lineal simple dada de acuerdo con las siguientes consideraciones:

$$
y_{i}=1.1337+0.0037 x
$$

$$
\begin{aligned}
& y_{i}=\text { Producto Interno Bruto } \\
& \beta_{0}=\text { Intercepto } \\
& \beta_{1}=\text { Pendiente calculada } \\
& x_{1}=\text { Deuda Pública }
\end{aligned}
$$

Donde:

Al respecto, se reconoce que el nivel del Producto Interno Bruto será de 1.1337 mil mdp cuando la deuda tenga un valor igual a cero. Mientras que en la medida que los saldos de la deuda gubernamental incrementen en una unidad, el producto interno bruto dispuesto a la cobertura de deuda incrementará en 0.0037 mil mdp, por lo cual implica un grado mayor de compromiso de la producción nacional para cobertura de pago.

El análisis de la relación de los Saldos de Deuda a tiempo, representa ser el foco de atención para este estudio, debido a que las variables tiempo y saldos de deuda, permiten comprender en ésta relación el riesgo financiero potencial en los Gobiernos locales. Debido a que dichos recursos económicos están destinados al pago de las Obligaciones

\section{La administración de la deuda pública de los gobiernos}


Financieras contratadas y determina su capacidad de pago ante la escasez de recursos propios originada por la baja recaudación fiscal.

c) Coeficientes de correlación y determinación de saldos de deuda a tiempo

Con base en los valores de los coeficientes de correlación y determinación que se describen en la tabla 6 , se puede observar que la correlación entre las variables saldos de deuda y tiempo en años presentan una $r=.9826$, que permite conocer la existencia de una correlación positiva muy alta. En donde ambas variables aumentan su valor simultáneamente.

Tabla 6. Resultados de los coeficientes de correlación y determinación

\begin{tabular}{cc}
\hline Tipo de coeficiente & Valor calculado \\
\hline Coeficiente de correlación $(r)$ & .9826 \\
\hline Coeficiente de determinación $\left(r^{2}\right)$ & .9655 \\
\hline Fuente: elaboración propia con base en SHCP (2018).
\end{tabular}

Y por otra parte el cálculo del coeficiente determinación entre dichas variables arrojo un valor de $\mathrm{r}^{2}=.9655$ significando que la proporción de la variación en $Y$ saldos de deuda se explica por efecto de $X$, es decir, los niveles de deuda aumentan en la medida en que se incrementa el tiempo, permitiendo explicar que el $96.55 \%$ de los incrementos de deuda se vieron afectados por las variaciones del tiempo transcurrido, según se describe en la figura 6 .

Dicho análisis representa ser un aspecto central para el presente estudio, debido a que la información obtenida permitió poner a prueba a la hipótesis de investigación. Cabe destacar que, el análisis de evaluación de la tendencia de la deuda pública consideró al periodo 2002 al 2017.

Esto se debió a que durante dicho intervalo de tiempo se presenta la administración de los Gobiernos neoliberales que implementaron la NGP y que en su momento promovieron las reformas administrativas y financieras enfocadas en contener los niveles desmedidos de deuda pública en las entidades Estatales y municipales. 
Figura 6. Correlación de las variables saldos de deuda y tiempo

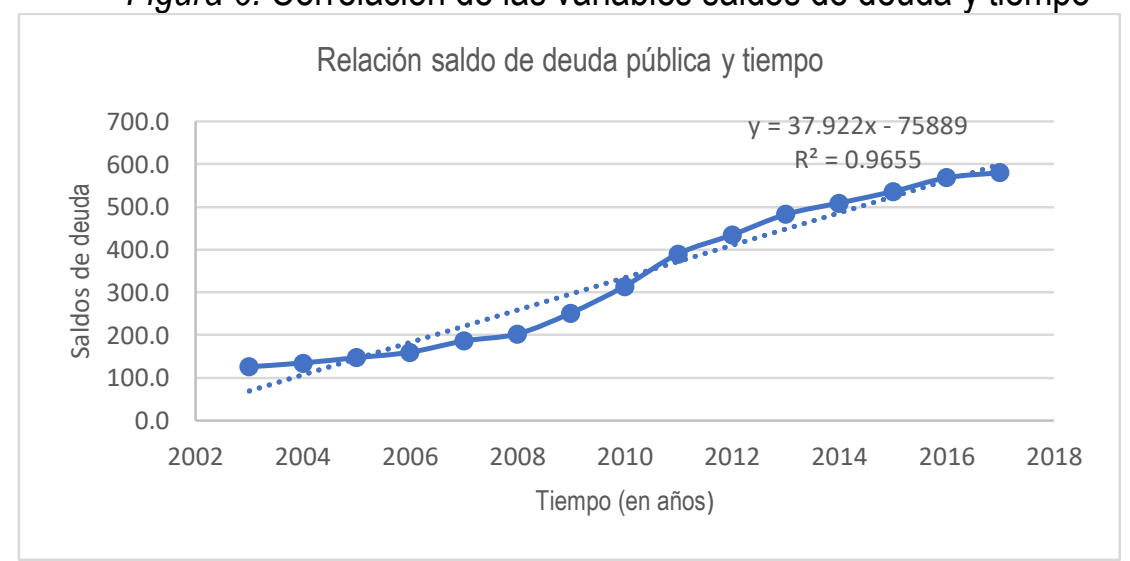

Fuente: Elaboración propia con base en SHCP (2018).

De acuerdo con la Figura 6. se presenta la ecuación de regresión lineal simple dada de acuerdo con las siguientes consideraciones:

$$
y_{i}=-75889+37.922 x
$$

$y_{i}=$ Saldo de la deuda

Donde:

$\beta_{0}=$ Intercepto

$\beta_{1}=$ Pendiente calculada

$x_{1}=$ tiempo en años

Al respecto, se reconoce que el nivel del saldo de la deuda será de 75.889 mil mdp cuando el tiempo en años tenga un valor igual a cero. Mientras que en la medida que el tiempo en años incrementen en una unidad, la deuda registrada incrementará en 37.922 mil mdp. Lo que implica que en la medida que el tiempo transcurre, el incremento de las obligaciones financieras también aumenta en los Gobiernos locales.

\section{Discusión}

El objetivo del presente estudio se centró en analizar el impacto de la implementación del modelo denominado la NGP en la administración de la

\section{La administración de la deuda pública de los gobiernos}


deuda pública y el riesgo financiero en los Gobiernos Locales de México. Con base en los fundamentos teóricos y en los resultados obtenidos a través del análisis descriptivo y correlacional, se pudo obtener suficiente evidencia empírica para aceptar la hipótesis de investigación formulada para el presente estudio, permitiendo en su momento conocer que la implementación de la NGP en la Administración Pública Estatal y municipal, ha traído como consecuencia el incremento desmedido de los niveles de las obligaciones financieras, poniendo en riesgo la sustentabilidad de las finanzas públicas en las entidades Estatales y municipales en México.

Uno de los principales indicadores a considerar en dicha conclusión, consistió en la relación tiempo y saldos de deuda, en donde se observó un incremento desmedido del nivel de las obligaciones financieras en diferentes cortes del tiempo, dando lugar a un mayor riesgo financiero en los Gobiernos locales. Comprometiendo significativamente a las Participaciones Federales del Ramo 28 poniendo en riesgo la capacidad de pago de las obligaciones financieras contraídas con los acreedores.

Durante dicho periodo de análisis de acuerdo con (Cejudo, 2011), la lógica administrativa de la NGP fue fuertemente criticada debido a sus efectos en la gestión cotidiana en los Gobiemos locales. Destacando que el problema administrativo central era la excesiva discrecionalidad en las funciones en los funcionarios públicos. De tal forma que en el ámbito nacional se cuestionó que, si bien era necesario un cambio en el sector público, las soluciones propuestas por la NGP no correspondían a los problemas concretos de la administración pública mexicana, la cual se caracteriza por contar con bajos estándares de rendición de cuentas, poco profesionalismo y poca legalidad. En este sentido Arellano (2002), indica que es indudable que administración pública debe cambiar vigilando su esencia y que la implementación de la lógica administrativa de la NGP está fuera de lugar ante las condiciones que encontramos en países como México.

Cabe señalar que la deuda pública no debe ser considerada como un factor negativo para los Gobiernos locales, siempre y cuando se administre observando y cumpliendo con el cúmulo de normas y reglamentos que se encuentran dentro del marco jurídico establecido en la Constitución Federal que regula formalmente la deuda pública, dentro de un marco de transparencia y de rendición de cuentas, enfocadas a fortalecer las finanzas

\section{Velázquez, S.}


públicas y las relaciones democráticas de los Gobiernos Locales con la ciudadanía.

Uno de los hallazgos más importantes en el análisis de la deuda pública en los Gobiernos locales en México, consiste en que el saldo total de la deuda al cuarto trimestre de 2017, se distribuye entre las entidades federativas de forma heterogénea, siendo las más endeudadas en términos absolutos, la Ciudad de México con 77.8 mil mdp, Nuevo León 71.2 mil mdp; Chihuahua 49.7 mil mdp; Veracruz, 49.1 mdp y el Estado de México con 45 mil 283.2 mil mdp. Estos cinco Estados concentran el 50.5\% del saldo total de la deuda pública de los Gobiernos locales que asciende a 580.6 mil mdp, según el registro único de obligaciones financieras (Secretaria de Hacienda y Crédito Público, 2018). Tema que representa ser una beta de investigación para otros investigadores.

\section{Referencias}

Aguilar, L. F. (2006). Gobernanza y Gestión Pública México. México: Fondo de Cultura Económica.

Anderson, D. R., Sweeney, D. J., \& Williams, T. A. (2008). Estadística para la Administración y Economía (10a.). México: Cenage.

Aregional. (2005). La deuda pública y calificaciones al riesgo crediticio de las entidades federativas. Recuperado http://aregional.com.mx/?target=buscador\&campo=La\%20Deuda\%20p\%FAblica\%2 Oy $\% 20$ calificaciones $\% 20$ al\%20riesgo\%20crediticio\%20de $\% 2010$ \% $\% 2$ municipios $\% 2$ Omexicanos

Arellano, D. (2002). Nueva gestión pública ¿el meteorito que mató a los dinosaurios? Reforma y democracia, 23 junio(Lecciones para la reforma administrativa en países como México).

Auditoria Superior de Fiscalización. (2017). Informe General del resultado de la Fiscalización Superior en Materia de Deuda Pública y Disciplina Financiera. Recuperado de http://www.orfis.gob.mx/CuentasPublicas/informe2017/archivos/TOMO\%20II/Volum en $\% 201 / 002 \% 20$ DEUDA\%20P\%C3\%9ABLICA\%20Y\%20DISCIPLINA\%20FINANC IERA\%20AL\%20EJERCITO\%202017.pdf

Auditoria Superior de la Federación. (2011). Análisis de la deuda Pública de las Entidades Federativas $y \quad$ Municipios. Recuperado de http://.asf.gob.mx/uploads/56_informes_especiales_de_auditoria/1._Analsis_de_De uda_publica_Agosto_2012

Cejudo, G. M. (2011). Nueva Gestión Pública. México: Biblioteca básica de Administración Pública.

\section{La administración de la deuda pública de los gobiernos}


Christensen, T., \& Leagreid, P. (2001). La Nueva Adminstración Pública: El equilibrio entre la gobernanza política y la autónomía administrtiva. Gestión y P'olítica Pública, X(1).

Diario Oficial de la Federación. (1995). Ley de Coordinación Fiscal de las Entidades Federativas $y$ los Municipios. Recuperado de http://www.diputados.gob.mx/LeyesBiblio/ref//cf/LCF_ref15_15dic95.pdf

Diario oficial de la Federación. (2016, abril 27). Ley de Disciplina Financiera de la Entidades Federativas y los Municipios. Reforma Adición y derogación de diversas disposiciones de las leyes de Coordinación Fiscal, General de Deuda Pública y General de Contabilidad Gubernamental. Recuperado de http://www.diputados.gob.mx/LeyesBiblio/ref/ldfefm.htm

García, I. M. (2007). La nueva gestión pública: Evolución y tendencias, presupuesto y gasto público. Secretaria General de Presupuestos y Gastos, Instituto de Estudios Fiscales, 47, 37-64.

Magallón, M. T. (2004). La implementación de la Nueva Gestión Pública a partir del enfoque Neoinstitucionalista: La eficiencia y la legitimidad como mitos. Universidad $\begin{array}{lll}\text { Autónoma } & \text { Metropolitana. } & \text { Recuperado } \\ \text { http://gestiónyestrategia.azc.uam.mx/index.php/rge/article//view/203 }\end{array}$

Magallón, M. T. (2008). Transferencia de Modelos Organizacionales propios del sector privado al sector público: El caso de la norma ISO 9000 en la CNDH. (Tesis Doctoral). Universidad Autónoma Metropolitana-Iztapalapa, México.

Secretaria de Hacienda y Crédito Público. (2018). Deuda Pública de Entidades Federativas y Municipios. Recuperado de https://www.gob.mx/hacienda

Torres, M. E., \& Juárez, M. A. (2016). La Nueva Gestión Pública en el contexto mexicano. Universidad Autónoma de Sinaloa. Recuperado de http://vertientepublicaeinternacional.mx/images/Numero2/ARTICULO-4.pdf 